\title{
Synthetic Stimuli for the Steady-State Verification of Modulation-Based Noise Reduction Systems
}

\author{
Jesko G. Lamm (EURASIP Member), Anna K. Berg, and Christian G. Glück
}

Bernafon AG, Morgenstrasse 131, 3018 Bern, Switzerland

Correspondence should be addressed to Jesko G. Lamm, Jesko.Lamm@vde-mitglied.de

Received 28 November 2008; Accepted 12 March 2009

Recommended by Heinz G. Goeckler

\begin{abstract}
Hearing instrument verification involves measuring the performance of noise reduction systems. Synthetic stimuli are proposed as test signals, because they can be tailored to the parameter space of the noise reduction system under test. The article presents stimuli targeted at steady-state measurements in modulation-based noise reduction systems. It shows possible applications of these stimuli and measurement results obtained with an exemplary hearing instrument.
\end{abstract}

Copyright (c) 2009 Jesko G. Lamm et al. This is an open access article distributed under the Creative Commons Attribution License, which permits unrestricted use, distribution, and reproduction in any medium, provided the original work is properly cited.

\section{Introduction}

Noise reduction systems provide users of hearing instruments with increased listening comfort [1]. The aim of such systems is to suppress uncomfortable sounds on the one hand, but to preserve speech on the other hand. Among various available noise reduction methods, modulationbased processing is a common one [2].

Modulation-based noise reduction systems apply different amounts of attenuation in different frequency ranges, depending on the likelihood of speech presence in each of them. Based on the observation that speech has a characteristic modulation spectrum [3], such systems measure modulation, which is the fluctuation of the signal's envelope over time.

The measurements treat modulation in different subbands separately, such that the signal processing can react by applying different amounts of attenuation in different frequency ranges. The idea is to attenuate signals that lack the characteristic modulation of speech.

Testing a hearing instrument regarding noise reduction performance has to ensure that two conditions are met.

(i) The noise reduction system meets its requirements.

(ii) The noise reduction system satisfies its user.

Assessing each of these conditions requires an individual test philosophy: while verification shows if the noise reduction system meets its requirements, validation assesses the system's capability of meeting customer needs "in the most realistic environment achievable" [4].

In the following, we present a measurement-based test procedure for modulation-based noise reduction systems in hearing instruments. Our focus is on verification and not on validation, because the validation of noise reduction systems in hearing instruments has been discussed well in literature (e.g., [5]).

Numerous stimulus-based verification procedures for different aspects of hearing instrument functionality have been presented so far. Here are two recent examples.

(i) The International Speech Test Signal $[6,7]$ is a stimulus for measuring the hearing instrument performance in a speech-like environment. It is based on a combination of numerous real-world speech signals.

(ii) Bentler and Chiou have discussed the verification of noise reduction systems in hearing instruments and presented measurements based on real-world speech in noise [8].

The above examples are both based on real-world speech signals. This makes sense, because suitable performance in speech is essential for hearing instruments. In this article, however, noise plays an important role, because noise is the reason why noise reduction systems are needed.

Real-world noise signals lack certain properties desirable in test signal design. These properties are mainly [9-11] 
(i) the possibility of configuring certain signal characteristics (like, e.g., modulation) systematically in order to force the system under test into a desired state;

(ii) freedom in changing the signal's temporal characteristics, selecting its power-spectrum, and making its spectral components sufficiently constant over the frequency range of interest.

We have therefore recently proposed synthetic test signals [9], because these can be synthesized with regard to the temporal characteristics of interest, the systematic estimation of noise reduction parameters, and accurate measurement results.

Using synthetic test signals in the audio processing domain is not new. In telephony applications, a so-called Composite Source signal [12] based on synthetic speech is available for verification of transfer characteristics of telephony equipment. In that case, again the speech performance of the system under test is of major interest, whereas the noise reduction stimuli which we describe in the following mainly focus on noise attenuation by the noise reduction system under test.

This article shows some new results and applications with the synthetic test signals from [9] in measuring the noise reduction attenuation in dependency of different input parameters. We first summarize and explain the signal synthesis procedure from [9]. Then we show different applications of the synthetic test signals. These are finally illustrated with measurement results, which we obtained with an exemplary hearing instrument.

\section{Synthetic Test Stimuli}

2.1. Requirements Towards the Stimuli. A noise reduction system should attenuate noise, which makes the attenuation a parameter of major interest in testing. Since a typical noise reduction system operates in multiple subbands [1], a systematic test procedure should measure the attenuation in each of them separately. It is therefore required that the test stimuli can stimulate each subband of the noise reduction individually and measure the impact on the system's frequency response.

As a consequence, the stimuli have to meet the following demands: they should not only perform well in frequency response measurements, but they should also allow signal parameters to be set individually for different frequency ranges. For example, the stimuli for verifying modulationbased noise reduction should have a constant magnitude in the frequency range of interest (see [11]) and furthermore different well-defined modulation depths in different frequency ranges.

The peak factor [13] of the signals should be as low as possible, because a high peak factor implies that a signal of given power has high amplitude peaks, resulting in distortion by nonlinearities not only in the measurement equipment but also in the hearing instrument under test itself.
The signals should also be periodic, since periodicity brings the following advantages.

(i) Periodic stimuli avoid leakage errors [14] in processing based on Discrete Fourier Transforms (DFT).

(ii) Measuring the magnitude of a system's frequency response becomes independent of the system's throughput delay when using a periodic stimulus, because within a given time frame whose length is an integer number of periods, the throughput delay of the system only produces a phase shift and thus has no impact on the measured magnitude.

(iii) Only one period of the desired stimulus needs to be computed, which limits synthesis time.

(iv) The stimulus can be described by means of its complex Fourier coefficients $\underline{c}_{k}$ via complex Fourier series. If the stimulus is $\sigma$, its period is $T$, and $j$ is the imaginary unit, then the Fourier Series representation of the stimulus is given by the following equation:

$$
\sigma(t)=\sum_{k=-\infty}^{\infty} \underline{c}_{k} \cdot e^{j 2 \pi(k t / T)} .
$$

Note that a disadvantage of a periodic signal is its discrete power-spectrum: measuring frequency responses with periodic stimuli will only cover discrete frequencies. See, for example, [14] for nonperiodic alternatives.

\subsection{Signal Synthesis}

2.2.1. Simple Subband Signals Based on Sinusoids. The most trivial subband signal is a sine wave. Rated against the requirements from Section 2.1, a sine wave performs well regarding peak factor and periodicity, but does not have the required constant magnitude over the frequency range of interest. This can be addressed in using multiple sines: a test stimulus obtained by summing sine waves of different frequencies will indeed cover a certain frequency range; however, summing sine waves requires special care, as will be explained below.

We call a sum of sine signals a multisine. Summing sine signals with carelessly chosen phase angles typically yields a multisine with a high peak factor [13] as opposed to the low peak factor required in Section 2.1. By choosing the right phase angles, the peak factor can be reduced: there are various algorithms for determining combinations of phase angles that yield a low peak factor in summing sine waves [13-16].

An exemplary multisine synthesis algorithm [15] has recently been evaluated in synthesizing stimuli for the verification of a noise reduction system in an exemplary hearing instrument [9]. Some poor results during this evaluation made us focus on noise-based stimuli, which will be discussed in the following. Note that it is still an open question whether multisines are a suitable basis for noise reduction stimuli, but we would like to exclude that question from this article's scope. 


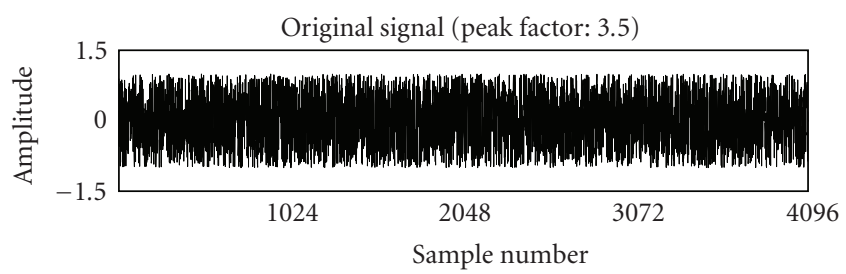

(a)

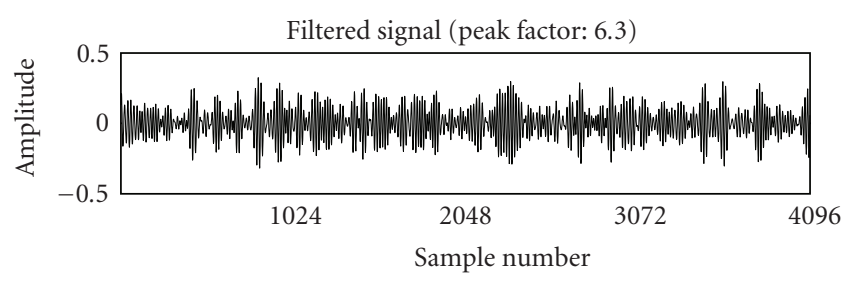

(b)

Figure 1: A noise signal (a) and the result of filtering it through a bandpass filter whose passband corresponds to a typical noise reduction subband (b).

2.2.2. Simple Subband Signals Based on Noise. A simple way of synthesizing a band-limited noise signal in the frequency range of a noise reduction subband is

(i) generating a noise signal,

(ii) filtering the noise signal with a bandpass filter whose pass-band corresponds to the noise reduction subband of interest.

Figure 1 shows an example: MATLAB's "rand" function was used to generate a uniformly distributed digital noise signal (Figure 1(a)). This signal was filtered with a bandpass filter corresponding to a typical noise reduction subband (Figure 1(b)).

However, the example in Figure 1 illustrates two reasons for why bandpass-filtering a noise signal will in general not produce a stimulus that meets the requirements from Section 2.1as follows.

(i) The stimulus has a high peak factor (e.g., a peak factor of 6.3 in the case of the signal shown in Figure 1(b)).

(ii) It is not possible to determine the modulation depth of the stimulus (see, e.g., the signal shown in Figure 1(b), which has some fluctuations in its envelope although it has not explicitly been modulated).

Obviously, subband signals that have been obtained by filtering a broadband signal are not well-suited as noise reduction stimuli. Therefore we propose to choose a synthesis procedure capable of constructing band-limited signals that have a low peak factor.

2.2.3. Band-Limited Discrete-Interval Binary Sequences. A signal whose amplitude has only two discrete values is called a binary signal. Obviously, binary signals have a minimum peak factor and therefore perfectly satisfy the peak factor requirement from Section 2.1. However, binary signals also tend to have a wide bandwidth, which disqualifies most of them from subband measurements.

The theory of discrete-interval binary signals [17, 18] provides algorithms that search binary signals with amplitude changes at multiples of a certain time interval for those signals whose power spectrum approximates a desired one. This theory can be used here to find binary signals whose power is concentrated in one subband and whose power spectrum is sufficiently constant for frequency response measurements.

Although binary signals have a minimum peak factor, it is not a necessary property for a noise reduction stimulus to be binary. We expect various kinds of signals to be suitable stimuli, like, for example, the multisines mentioned in Section 2.2.1. For our further considerations, however, we limit our scope to discrete-interval binary signals, because these performed well in our evaluation of different stimuli, as shown exemplarily in [9]. Since signal synthesis should work in discrete-time and discrete-interval binary signals were originally defined in continuous time [17], we define that a discrete-interval binary sequence is the discrete-time representation of a discrete-interval binary signal.

The Frequency Domain Identification Toolbox (FDIDENT) for MATLAB [19] offers readily accessible functions for synthesizing discrete-interval binary sequences [16]. The "dibs" function in this toolbox takes absolute values of desired Fourier coefficients as an input and returns one period of a periodic discrete-interval binary sequence whose Fourier series approximates the given input.

When used as a stimulus for subband measurements, a periodic discrete-interval binary sequence needs to have its power concentrated in the frequency range of interest, ideally like band-limited white noise. Therefore, if the frequency range of interest is from $f_{1}$ to $f_{2}$ (where $f_{1}>0$ and $f_{2} \geq f_{1}+T^{-1}$ ) and the desired RMS of the synthesized signal is $r$, then the target values for the synthesis algorithm are given by the following absolute values of Fourier coefficients $\underline{c}_{k}$ (derived from [9]):

$$
\begin{aligned}
& \left|\underline{c}_{k}\right| \\
& \quad= \begin{cases}\frac{r}{\sqrt{2\left(\left\lceil T \cdot f_{2}\right\rceil-\left\lfloor T \cdot f_{1}\right\rfloor+1\right)}} ; & \left.\mid T \cdot f_{1}\right\rfloor \leq|k| \leq\left\lceil T \cdot f_{2}\right\rceil \\
0 & \text { else. }\end{cases}
\end{aligned}
$$

\section{Frequency Response Measurements}

3.1. General Procedure. In this section, we describe a procedure for stimulus-based measurements of a linear system's frequency response. It is based on digital signal processing and thus assumes that test stimuli and the output signal of the system under test are available as digital waveforms. All further considerations will be made with regard to the following measurement procedure.

DFT-based processing can approximate the frequency response function of a system whose input stimulus is a periodic digital test signal: the system's output is digitized 
with the clock of the input signal. Then, the DFT is applied to both the input signal and the digitized output. The frequency response is calculated at each DFT frequency by dividing the absolute value of the output-related DFT bin by the corresponding input-related value $[11,20]$. Leakage errors can be avoided if the stimulus is periodic, and if the DFT window contains an integer number of its periods [14].

The described procedure requires a steady-state condition of the system under test. Therefore all measurements described here are steady-state measurements.

3.2. Subband Measurements with Narrow-Band Stimuli. A very simple test case is the measurement of the frequency response in one noise reduction subband. Let the number of subbands be $M$. We assign an index $i \in\{1,2,3, \ldots, M\}$ to each of them. Let the lower and upper band limit of band number $i$ be $f_{c, i}$ and $f_{c, i+1}$, respectively. Furthermore, let $u_{i}$ be a discrete-interval binary sequence that has been synthesized according to a target by (2), where $f_{1}=f_{c, i}$, and $f_{2}=f_{c, i+1}$.

We can now construct a signal with modulation frequency $f_{m, i}$ and a configurable modulation depth: let $m_{i}$ be another discrete-interval binary sequence that has been synthesized in the same way as $u_{i}$, but with $f_{1}=f_{c, i}+f_{m, i}$ and $f_{2}=f_{c, i+1}-f_{m, i}$, where the modification of the band limits by $\pm f_{m, i}$ should compensate for the broadened spectrum that results when modulating $m_{i}$ with modulation frequency $f_{m, i}$. Note that $m_{i}$ and $u_{i}$ are completely unmodulated. Now a signal $s_{i}$ of modulation frequency $f_{m, i}$ and configurable modulation depth is given by

$$
\begin{aligned}
s_{i}(t)= & \alpha \cdot \sqrt{\frac{2}{3}} \cdot m_{i}(t) \cdot\left[1+\cos \left(2 \pi f_{m, i} t\right)\right] \\
& +(1-\alpha) \cdot u_{i}(t) .
\end{aligned}
$$

In (3), parameter $\alpha \in[0,1]$ configures the modulation depth. The factor $\sqrt{2 / 3}$ ensures that the $s_{i}$ signals resulting from $\alpha=0$ and $\alpha=1$ have approximately the same RMS, such that the signal power of $s_{i}$ is almost independent of the modulation depth configured by parameter $\alpha$. In theory, the approximative factor $\sqrt{2 / 3}$ could be replaced by the precise factor that is needed to make the signal power completely independent from $\alpha$. This precise factor could be computed from the known signals $m_{i}$ and $u_{i}$. However, in the applications we present here, this is in our opinion not necessary: in the examples we show in this article based on the approximative factor $\sqrt{2 / 3}$, we computed the signal level of $s_{i}$ for the cases $\alpha=0$ and $\alpha=1$ and found that it differs by less than $0.05 \mathrm{~dB}$ from one case to the other.

Note that the stimuli we present here are defined in continuous time, but targeted at a measurement procedure using discrete-time processing. The link between the continuoustime domain and the discrete-time domain is in our case given by the earlier-mentioned FDIDENT toolbox: It takes Fourier coefficients from the continuous-time domain as an input and returns a discrete-interval binary sequence as discrete-time signal. Therefore, the following discrete-time version of (3) is needed (where $n$ is the sample index, $f_{s}$ is the sampling frequency and $\hat{m}_{i}, \hat{u}_{i}, \hat{s}_{i}$ are the discrete-time signals resulting from sampling $m_{i}, u_{i}$, and $s_{i}$, resp.):

$$
\begin{aligned}
\hat{s}_{i}(n)= & \alpha \cdot \sqrt{\frac{2}{3}} \cdot \hat{m}_{i}(n) \cdot\left[1+\cos \left(2 \pi \frac{f_{m, i}}{f_{s}} n\right)\right] \\
& +(1-\alpha) \cdot \hat{u}_{i}(n) .
\end{aligned}
$$

The signal from (4) can be used for measuring the frequency response of a hearing instrument in the subband of interest with the procedure from Section 3.1. The frequency response of the noise reduction system in the hearing instrument can be obtained by a differential measurement; this means that the frequency response is first measured with the noise reduction turned on and then with the noise reduction switched off. Frequency-by-frequency division of the obtained responses yields the transfer function of the noise reduction.

Note that the signal presented in this section is only targeted at a single subband. Therefore all measurement samples at frequencies outside the given subband have to be ignored. The next section describes stimuli that measure the frequency response of the noise reduction system over the whole bandwidth of the hearing instrument.

\subsection{Full Bandwidth Measurements with Broadband Stimuli.}

This section describes the synthesis of a signal that allows measuring the frequency response over the whole bandwidth of the hearing instrument under test. The idea is to measure the effect of the noise reduction system in a certain subband, while the noise reduction does not act on any other frequency range. Let the subband of interest be $b$. To obtain a test signal $\theta_{b}$ that evokes attenuation of a modulation-based noise reduction system in subband number $b$ only, we add a signal that is designed with configurable modulation and limited to have most of the signal power in subband number $b$ to fully modulated signals corresponding to the other subbands:

$$
\begin{aligned}
\theta_{b}(n)= & \hat{s}_{b}(n)+\sqrt{\frac{2}{3}} \cdot \sum_{i \in(\{1,2, \ldots, M\} \backslash\{b\})} \hat{m}_{i}(n) \\
& \cdot\left[1+\cos \left(2 \pi \frac{f_{m, i}}{f_{s}} n\right)\right] .
\end{aligned}
$$

Here again, $M$ is the number of subbands. The signal $\widehat{s}_{b}$ in (5) is the same as in (4). This means that the modulation depth of signal $\hat{s}_{b}$ can be configured via parameter $\alpha$ according to (4).

Note the following: if the value of $\alpha$ is close to 1 , some segments of signal $\hat{s}_{b}$ are close to zero (those segments in which the cosine in (4) is close to -1$)$. As the $\hat{m}_{i}$ signals in (5) are discrete-interval binary sequences, they will not be perfectly band-limited and therefore produce side-lobes in subband number $b$. This means that the stimulus in the subband of interest is infringed by sidelobes from other bands for values of $\alpha$ close to 1 .

As a consequence, the test signal $\theta_{b}$ from (5) is not well-suited for measuring noise reduction performance as a function of modulation depth parameter $\alpha$ in its full range. 
However, for $\alpha=0$, signal $\theta_{b}$ can be used for measuring the frequency response of the noise reduction system while one subband is stimulated to apply its maximum attenuation. We show an example of this application in Section 5.4.1.

3.4. Subband Measurements with Broadband Stimuli. So far we have presented the synthesis of noise reduction stimuli for different subbands and a way of mixing these stimuli in order to obtain a test signal $\theta_{b}$ for broadband measurements. We argued that test signal $\theta_{b}$ causes problems with high modulation depths due to side-lobe influences from other subbands. In this section we show a way of eliminating these side-lobe influences in one subband of interest.

If subband number $b$ is the subband of interest, then we can eliminate the influences from other subbands by filtering out their side lobes from this particular subband. This can be done using a band-stop filter whose band limits are the crossover frequencies of subband number $b$. Let $h$ be the impulse response of such a band-stop filter, and let “*” be the convolution operator. Furthermore let $\tilde{\theta}_{b}$ be a modified version of $\theta_{b}$ in which side-lobe influences from other subbands will be eliminated. We construct $\widetilde{\theta}_{b}$ by a modified version of (5)

$$
\begin{aligned}
\tilde{\theta}_{b}(n)= & \hat{s}_{b}(n)+\sqrt{\frac{2}{3}} \cdot h(n) * \sum_{i \in(\{1,2, \ldots, M\} \backslash\{b\})} \hat{m}_{i}(n) \\
& \cdot\left[1+\cos \left(2 \pi \frac{f_{m, i}}{f_{s}} n\right)\right] .
\end{aligned}
$$

In practice, we do not implement the band-stop filter by a convolution with $h$. We rather implement the filtering in the DFT domain: we put zeros into the stop-band's DFT bins of the signal to filter, exploiting the periodicity of the $\hat{m}_{i}$ signals and of the cosine term in (6).

Note that $\tilde{\theta}_{b}$ can have a higher peak factor than $\theta_{b}$ due to the filtering. In the measurements we describe in Section 5.4.2, this was however not a problem. If only one subband of interest is within the scope of the test, then the narrow-band stimuli from Section 3.2 can be used. The test signal $\widetilde{\theta}_{b}$ is useful when all subbands of the noise reduction system are relevant in the test case, but modulation will only be varied in one of them.

\section{Attenuation Function Measurements}

Modulation-based noise reduction systems apply attenuation as a function of the signal's modulation depth [8]. Therefore, the dependency between modulation depth and attenuation is of interest in noise reduction testing. For systems that operate in multiple subbands, this dependency can be assessed per subband, if varying modulation parameter $\alpha$ according to (4) and then using each resulting signal $\hat{s}_{i}$ either as a stimulus for measurement or as a basis for synthesizing stimulus $\tilde{\theta}_{b}$ according to (6).

The resulting stimuli can be used in measuring the frequency response of a subband of interest for different modulation depths. In order to obtain a simple modulation/attenuation dependency function, one needs to compute a single attenuation value from a transfer function defining gain at multiple frequencies. Inspired by the way in which median and averaging operations work, we here propose sorting a certain set of gain values within the subband of interest by their magnitude and then averaging those values that remain after eliminating the first and the last quarter of the resulting sorted list. Typically, one would only choose frequencies close to the center frequency of the current subband in order to avoid taking the slopes at the band limits into the averaging process.

\section{Examples}

5.1. System under Test. An exemplary digital hearing instrument with a modulation-based noise reduction system was the system under test for the measurements whose results are presented further below. The noise reduction system in this hearing instrument works in the time domain according to the following scheme.

(i) Determine the amount of typical modulation in different subbands of the hearing instrument's input signal by passing subband signals through running maximum and minimum filters and comparing the different filters' outputs [1].

(ii) For each subband, compute attenuation as a function of modulation, where low modulation maps to high attenuation and vice versa.

(iii) Use a controllable filter to adjust the frequency response of the hearing instrument as it is given by the computed frequency-dependent attenuation values.

More details on the underlying concept of implementing modulation-based noise reduction in the time domain can be found in [1].

5.2. Measurement Setup. A test system was set up for making measurements with synthetic test signals. Figure 2 illustrates the setup: the hearing instrument under test is located in an off-the-shelf acoustic measurement box with a loudspeaker $\left(L_{1}\right)$ for presenting test stimuli to be picked up by the hearing instrument's input transducer $\left(M_{2}\right)$. The hearing instrument's output transducer $\left(L_{2}\right)$ is coupled with a measurement microphone $\left(M_{1}\right)$ so tightly that environment sounds can be neglected in comparison to the hearing instrument's output. The coupler is a cavity that is similar to the human ear canal. Here, we used a so-called 2cc-coupler.

A digital playback and recording system can play MATLAB-created stimuli via a digital-to-analogue converter (D/A) and the loudspeaker of the measurement box $\left(L_{1}\right)$, while recording the hearing instrument's output via the measurement microphone and an analogue-to-digital converter $(\mathrm{A} / \mathrm{D})$. The recorded digital data is stored in a MATLABreadable file on a hard disk. The sampling rate for both playing and recording signals is $22050 \mathrm{~Hz}$. The test system ensures synchronous playback and recording. 
5.3. Measurement Procedure. The gain in the hearing instrument under test was set $20 \mathrm{~dB}$ below the maximum offered value to reduce nonlinearities. All adaptive features of the hearing instrument, apart from noise reduction, were turned off for all test runs. The hearing instrument was furthermore configured for linear amplification; this means that there was no dynamic range compression.

Measurements were performed with different $\theta_{b}$ and $\tilde{\theta}_{b}$ according to (5) and (6), respectively. In synthesizing these $\theta_{b}$ and $\tilde{\theta}_{b}$, the required $\hat{m}_{i}$ and $\hat{u}_{i}$ were computed by function "dibs" of the earlier-mentioned FDIDENT toolbox, and synthesis parameter $r$ in (2) was adjusted to yield a $70 \mathrm{~dB}$ SPL level in each subband. Our measurement method foresees the use of different values of the band index $b$. However, for simplicity, one constant $b$ was exemplarily chosen for all measurements we present here.

The DFT-based processing according to Section 3.1 was used for frequency response measurements. As this processing needs an integer number of stimulus periods to fit into a DFT window, we chose the stimulus period to be equal to the DFT window length: a window length of 4096 samples allowed us both the use of the Fast Fourier Transform (FFT) and the choice of about $5.4 \mathrm{~Hz}$ modulation frequency $\left(f_{m}=\right.$ $f_{s}$ /window length in samples). This frequency is typical for speech, whose modulation spectrum is significant in the range from 1 to $12 \mathrm{~Hz}$ [3].

Two experiments were performed per stimulus: first with the noise reduction system of the hearing instrument switched off, and second while having it switched on. This allowed us to achieve the differential measurement that has been mentioned in Section 3.2: instead of comparing the output and input signal of the system, we compared the output signal from the second experiment with the one from the first experiment.

This method made the measurement procedure independent of the throughput delay in the system under test, especially because the throughput delay of the system was much smaller than the stimulus duration and therefore negligible for test timing; this means that we did not need to delay the recording of output signals compared to the playback of input stimuli. Note that even variations in throughput delay between the first and second experiment could not influence the result, because magnitude computations were independent of the throughput delay due to the periodicity of the used stimuli (see Section 2.1).

\subsection{Measurement Results}

5.4.1. Frequency Response of an Exemplary Noise Reduction System. We measured the frequency response of the noise reduction system under test while high attenuation was required in one subbband and no attenuation was required in the other ones. In order to trigger this noise reduction behavior on the one hand and to allow a measurement over the whole bandwidth of the system on the other hand, we used signal $\theta_{b}$ according to (5) as a stimulus, where parameter $\alpha$ in the synthesis of signal $\hat{s}_{b}$ via (4) was set to zero.

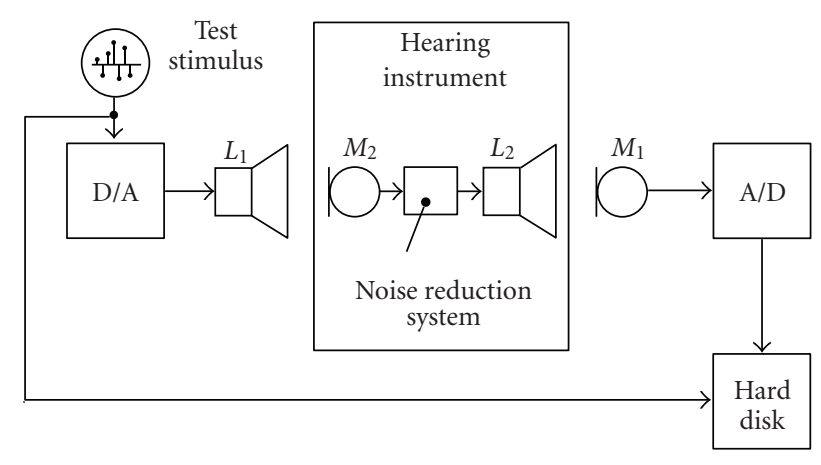

Figure 2: Measurement setup.

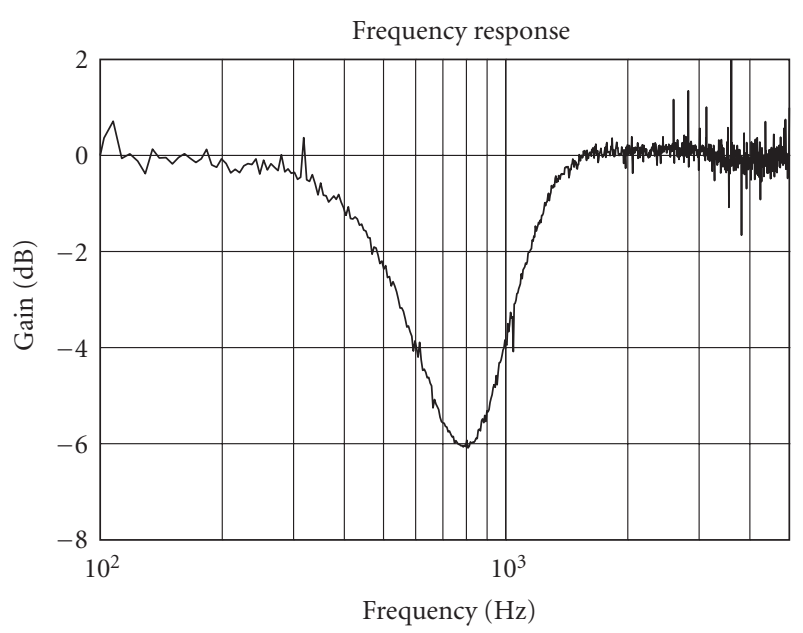

Figure 3: Measured frequency response of a noise reduction system that is stimulated for attenuating one subband.

For each measurement, the test stimulus was presented during 15 seconds in order to allow the system under test to reach steady state. Bin-by-bin division of FFT absolute values from the second measurement by corresponding values of the first measurement delivered the frequency response of the noise reduction system. Five FFT windows were averaged for spectral smoothing [21]. These windows were taken from the last five seconds of the test run in order to observe the steadystate condition.

The result of this measurement is shown in Figure 3: the shown frequency response indicates that the noise reduction system under test provides attenuation in a subband around $800 \mathrm{~Hz}$ while not attenuating any other subband.

\subsubsection{Attenuation Function of an Exemplary Noise Reduction} System. We measured the dependency of attenuation on modulation depth, where we defined that attenuation is the average over the transfer function samples at frequencies in a band of $100 \mathrm{~Hz}$ around the center frequency of the subband of interest. One can argue that the narrow-band signal $\hat{s}_{i}$ from (4) is the suitable stimulus for this kind of measurement. However in our case, we based our stimulus on $\widetilde{\theta}_{b}$ from (6) in order to have a broadband stimulus 


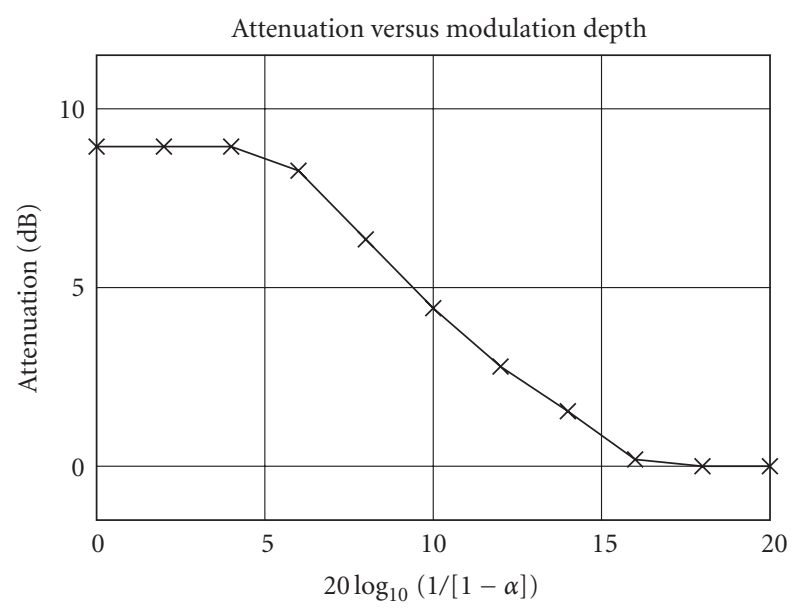

Figure 4: Measured dependency of noise reduction attenuation on modulation depth parameter $20 \cdot \log _{10}\left([1-\alpha]^{-1}\right)$.

with constant signal properties in most of the subbands. This is an advantage when testing hearing instruments with environment-dependent automatics, because the constant subband signals we have in $\tilde{\theta}_{b}$ represent a defined environment, whereas $\hat{s}_{i}$ does not impose a defined environment on any subband but the one of interest.

Measurements were performed with a modified version of $\tilde{\theta}_{b}$. The modification was to choose a sum index range of $(\{1,2, \ldots, M\} \backslash\{b-1, b, b+1\})$ instead of $(\{1,2, \ldots, M\} \backslash$ $\{b\})$ from (6). This modification ensured that the nominal frequency range of the band of interest spanned more than one subband of the noise reduction system under test, thus making the procedure invariant to clock differences between the device under test and test equipment and to nonideal subband split.

For the same reason, the band-stop filtering that is part of (6) was made with a band-stop filter whose band limits were set far enough outside the subbband of interest to reach further than the filter slopes of the corresponding subband split filter. We had to find a compromise between setting the band limits of the band-stop filtering far outside the subband of interest to make the measurement procedure robust and setting them as close as possible to these band limits in order to keep signal changes by the filtering as little as possible. One reason to be careful with the choice of the stop-band filter design is that the filtering can change the peak factor of the synthesized stimuli (see also Section 3.4). As a result, we chose band-stop corner frequencies that were half a subband width away from the band limits of the subband of interest.

We used different test stimuli that resulted from varying modulation parameter $\alpha$ according to (4). Since parameter $\alpha$ is in the amplitude domain, whereas usual hearing instrument specifications use decibels as unit, we used $20 \cdot \log _{10}\left([1-\alpha]^{-1}\right)$ rather than $\alpha$ as the modulation depth parameter.

We varied $20 \cdot \log _{10}\left([1-\alpha]^{-1}\right)$ in steps of 2 and measured the noise reduction attenuation as a function of this varied parameter. The measured frequency response that was used as a basis for computations was smoothed by averaging over five FFT windows [21].

To obtain one single attenuation value from the frequency response of interest, the proposed procedure from Section 4 was used; this means that the frequency response was searched for gain values corresponding to frequencies in $\mathrm{a} \pm 50 \mathrm{~Hz}$ range around the center frequency of the subband of interest, and the corresponding gain values were then sorted and finally averaged after eliminating the first and last quarter of the sorted list.

The obtained result is shown in Figure 4. We see that the system under test behaves as one would expect of a modulation-based noise reduction system (e.g., [8]): Unmodulated signals are attenuated strongly, whereas modulated signals are not attenuated or attenuated less strongly.

\section{Conclusion}

Synthetic test signals have been proposed for verification in the domain of digital hearing instruments. Discreteinterval binary sequences have been used to synthesize stimuli targeted at systematic verification of a modulationbased noise reduction system.

Measurements with an exemplary hearing instrument showed that the synthetic signals succeeded in both stimulating the noise reduction in the subband of interest and measuring the system's frequency response and attenuation function. With the given stimuli, it is possible to test against specifications that require noise reduction attenuation as a function of frequency and modulation.

\section{References}

[1] A. Schaub, Digital Hearing Aids, Thieme Medical, New York, NY, USA, 2008.

[2] V. Harnacher, J. Chalupper, J. Eggers, et al., "Signal processing in high-end hearing aids: state of the art, challenges, and future trends," EURASIP Journal on Applied Signal Processing, vol. 2005, no. 18, pp. 2915-2929, 2005.

[3] I. Holube, V. Hamacher, and M. Wesselkamp, "Hearing instruments-noise reduction strategies," in Proceedings of the 18th Danavox Symposium on Auditory Models and Nonlinear Hearing Instruments, pp. 359-377, Kolding, Denmark, September 1999.

[4] A. Kossiakoff and W. N. Sweet, Systems Engineering Principles and Practice, John Wiley \& Sons, Hoboken, NJ, USA, 2003.

[5] M. Marzinzik and B. Kollmeier, "Predicting the subjective quality of noise reduction algorithms for hearing aids," Acta Acustica united with Acustica, vol. 89, no. 3, pp. 521-529, 2003.

[6] I. Holube, "Development and analysis of an international speech test signal (ISTS)," in International Hearing Aid Research Conference (IHCON '08), Lake Tahoe, CA, USA, August 2008.

[7] I. Holube and EHIMA-ISMADHA Working Group, "Short description of the international speech test signal (ISTS)," EHIMA-European Hearing Instrument Manufacturers Association, (document contained in a download package with the ISTS signal, April 2008), June 2007, http://www.ehima.com. 
[8] R. Bentler and L.-K. Chiou, "Digital noise reduction: an overview," Trends in Amplification, vol. 10, no. 2, pp. 67-82, 2006.

[9] J. G. Lamm, A. K. Berg, and C. G. Glück, "Synthetic signals for verifying noise reduction systems in digital hearing instruments," in Proceedings of the 16th European Signal Processing Conference (EUSIPCO '08), Lausanne, Switzerland, August 2008.

[10] P. E. Wellstead, "Pseudonoise test signals and the fast fourier transform," Electronics Letters, vol. 11, no. 10, pp. 202-203, 1975.

[11] H. A. Barker and R. W. Davy, "System identification using pseudorandom signals and the discrete fourier transform," Proceedings of the IEE, vol. 122, no. 3, pp. 305-311, 1975.

[12] H. W. Gierlich, "New measurement methods for determining the transfer characteristics of telephone terminal equipment," in Proceedings of the IEEE International Symposium on Circuits and Systems (ISCAS '92), pp. 2069-2072, San Diego, CA, USA, May 1992.

[13] M. R. Schroeder, "Synthesis of low-peak-factor signals and binary sequences with low autocorrelation," IEEE Transactions on Information Theory, vol. 16, no. 1, pp. 85-89, 1970.

[14] R. Pintelon and J. Schoukens, System Identification: A Frequency Domain Approach, IEEE Press, New York, NY, USA, 2001.

[15] E. Van der Ouderaa, J. Schoukens, and J. Renneboog, "Peak factor minimization using a time-frequency domain swapping algorithm," IEEE Transactions on Instrumentation and Measurement, vol. 37, no. 1, pp. 145-147, 1988.

[16] K. R. Godfrey, A. H. Tan, H. A. Barker, and B. Chong, "A survey of readily accessible perturbation signals for system identification in the frequency domain," Control Engineering Practice, vol. 13, no. 11, pp. 1391-1402, 2005.

[17] A. van den Bos and R. G. Krol, "Synthesis of discrete-interval binary signals with specified fourier amplitude spectra," International Journal of Control, vol. 30, no. 5, pp. 871-884, 1979.

[18] K.-D. Paehlike and H. Rake, "Binary mutifrequency signalssynthesis and application," in Proceedings of the 5th IFAC Symposium on Identification and System Parameter Estimation, vol. 1, pp. 589-596, Darmstadt, Germany, September 1979.

[19] I. Kollár, Frequency Domain System Identification Toolbox V3.3 for Matlab, Gamax Ltd, Budapest, Hungary, 2004.

[20] S. T. Nichols and L. P. Dennis, "Estimating frequency-response function using periodic signals and the f.f.t," Electronics Letters, vol. 7, no. 22, pp. 662-663, 1971.

[21] J. S. Bendat and A. G. Piersol, Random Data: Analysis and Measurement Procedures, John Wiley \& Sons, New York, NY, USA, 2000. 